\title{
CD Methods Development at the Bio-Nano Interface
}

\author{
Andreas Hennig, Sheshanath Bhosale ${ }^{a}$ Naomi Sakai, and Stefan Matile*
}

\begin{abstract}
We describe recent applications of circular dichroism (CD) spectroscopy to develop simple methods for the study of supramolecular functional materials. Examples include the development of a CD method to identify supramolecules in action (and to determine Hill coefficients for exergonic self-assembly), or CD methods to measure osmotic pressure and the activity of ion channels and pores. For the latter two applications, G-quartets are introduced as versatile functional CD probes in chirogenic vesicles.
\end{abstract}

Keywords: Circular dichroism spectroscopy $\cdot$ Ion channel · Osmotic pressure $\cdot$ Pores $\cdot$ Supramolecular function

In our research that focuses on the creation of functional supramolecules (ion channels, pore sensors, photosystems), ${ }^{[1-6]}$ chirality is usually not a main subject, but is used as a powerful tool. ${ }^{[7]}$ For example, experimental evidence for the desired function is often backed up with corroborative structural studies by circular dichroism(CD) spectroscopy under meaningful conditions. Topics related to chirality such as the detection of enantiomeric excess with synthetic pores are delightful exceptions to this rule. ${ }^{[8]}$ However, CD studies can attract full attention when new methods are needed to address new questions. Such adventures off the beaten track will be summarized briefly in the following. Shifting the focus of at-

${ }^{*}$ Correspondence: Prof. S. Matile Department of Organic Chemistry University of Geneva

Quai Ernest-Ansermet 30

$\mathrm{CH}-1211$ Geneva 4

Tel.: + 41223796523

Fax: + 41223793215

E-mail: stefan.matile@chiorg.unige.ch

aCurrent address: Department of Chemistry

Monash University

Clayton, Australia tention from the usual detection of structure using CD spectroscopy to the less explored detection of function, we first describe recent CD methods development to measure osmotic pressure ${ }^{[9]}$ and the activity of ion channels and pores. ${ }^{[10]}$ We further include the development of a simple CD method to identify supramolecules in action (and to determine denaturation-corrected Hill coefficients for exergonic self-assembly), ${ }^{[11]}$ and en passant also the CD silencing associated with helix-barrel transition. ${ }^{[12]}$

A rich collection of $\mathrm{CD}$ methods for structural studies has been developed over the last few decades. This covers routine determination of absolute configuration and enantiomeric purity, conformational analysis including folding, programmed assembly, ${ }^{[13]}$ the formation of host-guest complexes, and more. ${ }^{[14]}$ Compared to this everyday use in structural studies, the use of CD spectroscopy to study function is clearly less developed. This seems regrettable because advantages such as sensitivity and selectivity for chiral probes would be similarly attractive. Moreover, the perspective to use ECCD doublets to eliminate false positives by ratiometric detection appears as appealing as the possible combination with achiral fluorescence probes for simultaneous detection in double-channel $\mathrm{CD}$ / fluorescence kinetics. ${ }^{[10]}$

Osmotic stress provided a valuable target for CD methods development to detect function. [9] For this purpose, G-quartets were introduced as functional CD probes. G-Quartets are planar supramolecular macrocycles of four guanosines around a central potassium cation (Fig. 1). To detect osmotic pressure, lipid bilayer vesicles are loaded with 5'-guanosine monophosphate (GMP)
1 and potassium cations at concentrations needed for the partial formation of G-quartets 2 . These G-quartets are reported in the CD spectrum as a ECCD doublet with negative $\mathrm{CD}$ amplitude centered around 255 nm (Fig. 2A). The amplitude of this ECCD doublet decreases in response to external under- and increases in response to external overpressure. This decrease of ECCD is in agreement with G-quartet disassembly as a result of vesicle swelling under hypoosmotic conditions. The complementary ECCD increase under hyperosmotic conditions confirms G-quartet assembly in response to internal volume decrease by vesicle shrinking. Representative dose response curves at low and high potassium concentrations demonstrate that osmotic pressure is readily detectable by CD spectroscopy (Fig. 2B). Variation of the concentration of potassium cations or GMP allows finetuning of the assay to target specific, hypoosmotic or hyperosmotic ranges of detection.

The response of intravesicular G-quartets to osmotic pressure is of particular interest with regard to the crawling of animal cells. It demonstrates that internal disassembly as response to hyperosmotic conditions to counteract external overpressure and relief bilayer stress does not occur, at least not in this simple form.

The use of G-quartets as functional CD probes is obviously not limited to the detection of osmotic stress. One of the simplest applications is the detection of the activity of pores that can mediate GMP efflux. ${ }^{[10]}$ The resulting local GMP dilution will cause G-quartet disassembly. As a result, pore activity will be reported as CD silencing (Fig. 3A). Validity of this CD approach to study pores was demonstrated using melittin, a 


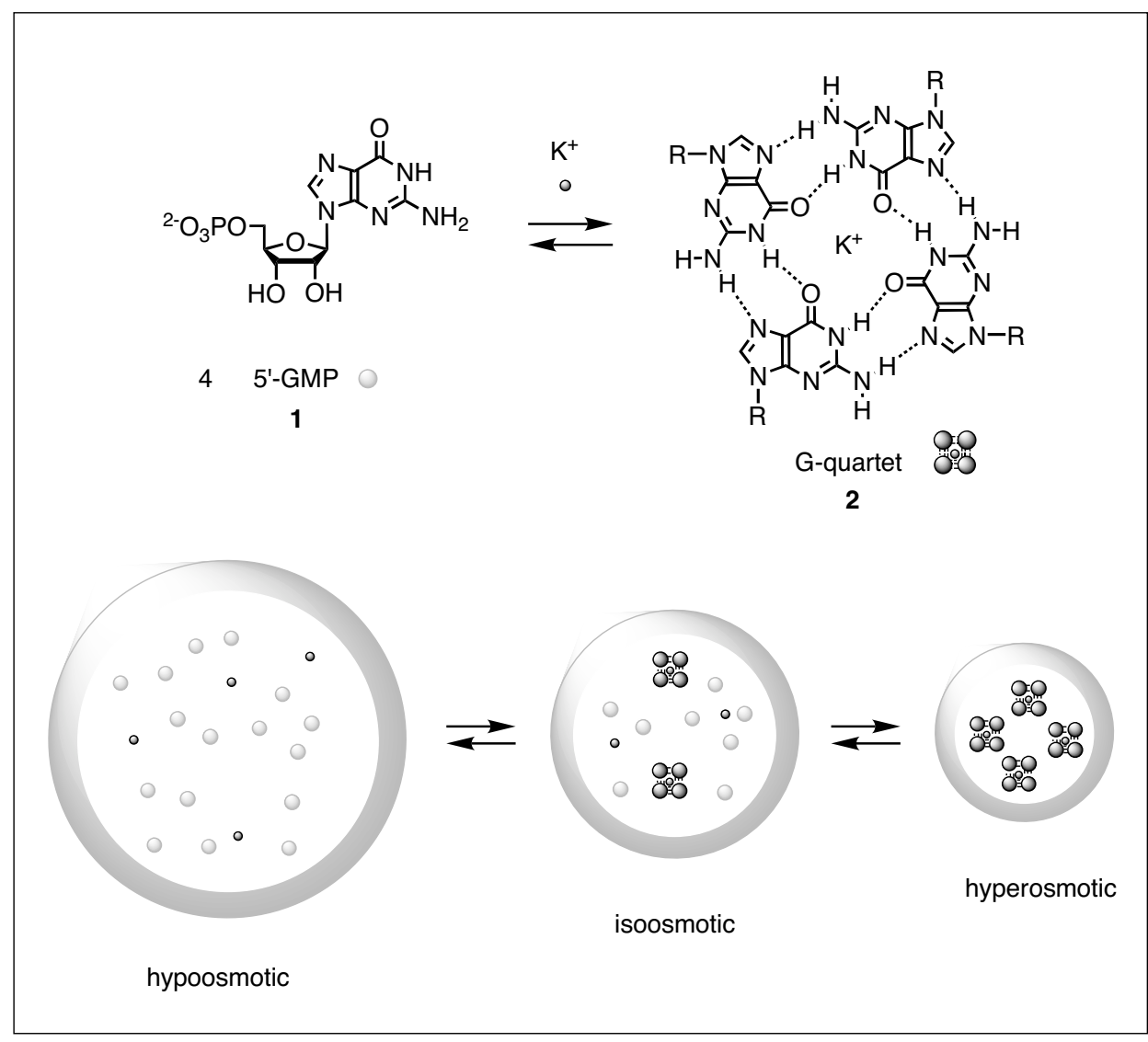

Fig. 1. Potassium-templated self-assembly of 5'-guanosine monophosphate (GMP) 1 into G-quartets 2. Continuing self-assembly into face-to-face $\pi$-stacked octamers and longer columnar stacks is not shown. Encapsulated as mixture of monomers $\mathbf{1}$ and tetramers $\mathbf{2}$ in a vesicle under isoosmotic conditions, application of osmotic overpressure results in GMP assembly because of vesicle shrinking, whereas application of osmotic underpressure results in G-quartet disassembly because of vesicle swelling.
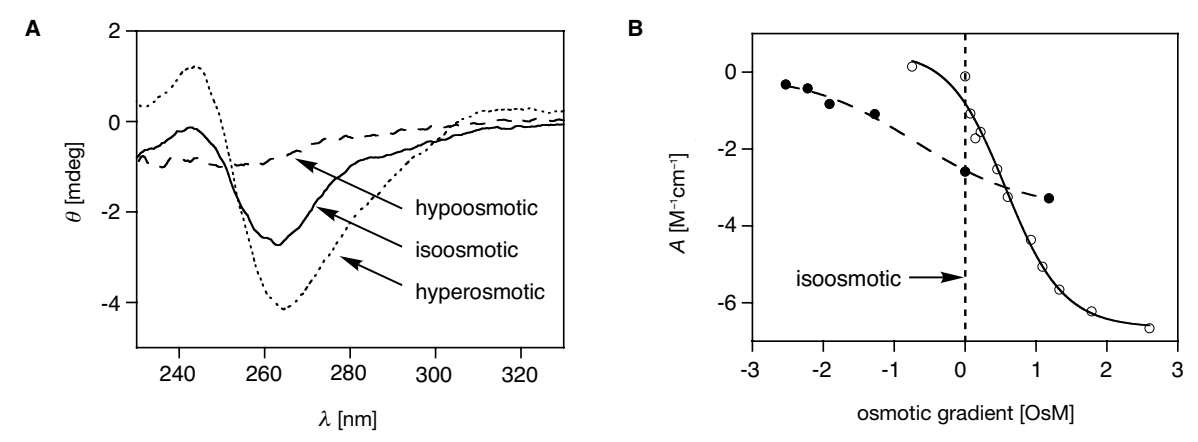

Fig. 2. (A) CD spectra of GMP 1 within lipid bilayer vesicles under isoosmotic (solid), hypoosmotic (dashed) and hyperosmotic (dotted) conditions, and (B) dependence of the CD amplitude A on osmolarity at constant GMP and low (open circles) and high (filled circles) potassium concentrations. Reproduced with permission from ref [9], () 2003 Wiley-Liss, Inc.

major component of honeybee venom, as a model pore. The same is naturally true for the detection of the activity of anion carriers/transporters that are capable to transport GMP across bilayer membranes. Facile detectability of the activity of counteranionactivated cell-penetrating peptides such as dodecylphosphate-polyarginine complexes by CD spectroscopy reveals that these synergistic anion transporters are capable to transport GMP across vesicle membranes. tion. Namely, we first load vesicles with G-quartets in the presence of the favorable potassium cation template. Addition of an ion channel turns on potassium cation exchange across the bilayer membrane. This event passes unnoticed by CD spectroscopy because the concentration of intravesicular G-quartets does not change. However, replacement of external potassium cations by cesium cations changes this situation (Fig. 3B). Addition of ion channels now initiates transmembrane exchange of potassium and cesium cations to make the small amount of the former vanish in the large extravesicular space and leave vesicles filled with cesium behind. This internal exchange of the favorable potassium cations with the unfavorable cesium cations causes the G-quartets to disassemble. Ion channel activity thus results in CD silencing (Fig. 4A).

Reversal of the system makes the chiroptical detection of ion channel activity even more attractive because activity is reported as appearance rather than disappearance of a CD signal. For this purpose, vesicles are loaded with GMP in the presence of cesium cations at concentrations insufficient for Gquartet formation (Fig. 3C). The addition of ion channels allows external potassium to enter the vesicle and template the formation of G-quartets. As a result, ion channel activity is reported as chirogenesis (Fig. 4B). The resulting CD signal can be easily silenced by lysis. This chirogenic detection of ion channels is more satisfactory than CD silencing because it operates only in intact vesicles. Remaining with gramicidin $\mathrm{A}$ as example, the detection of ion channel activity with CD spectroscopy turns out to be compatible with the determination of Hill coefficients and ion selectivity topologies. Both gramicidin $\mathrm{A}(n \approx 2)$ and melittin $(n \approx$ 4) are $n>1$ systems with unstable active suprastructures; the controversies surrounding exhaustive structural studies on both model channels underscore the fundamental importance to understand the dilemma of Hill analysis applied to self-assembly that will be described in the following.

In general, one key characteristic of supramolecular functional architecture is cooperativity: More than one molecule is needed to produce an active structure that can accomplish significant function. ${ }^{[11]} \mathrm{Co}-$ operativity is traditionally expressed with the Hill coefficient $(n)$. The Hill equation describes the dependence of a signal $S$, reporting on structure or activity, on the n-th power of the concentration $c_{\mathrm{M}}$ of the active monomer, i.e.

$$
S \propto\left(c_{\mathrm{M}} / E C_{50}\right)^{n}
$$

A Hill coefficient $n>1$ demonstrates cooperativity or supramolecular function, whereas Hill coefficient $n=1$ demonstrates unimolecular function without coopera- 


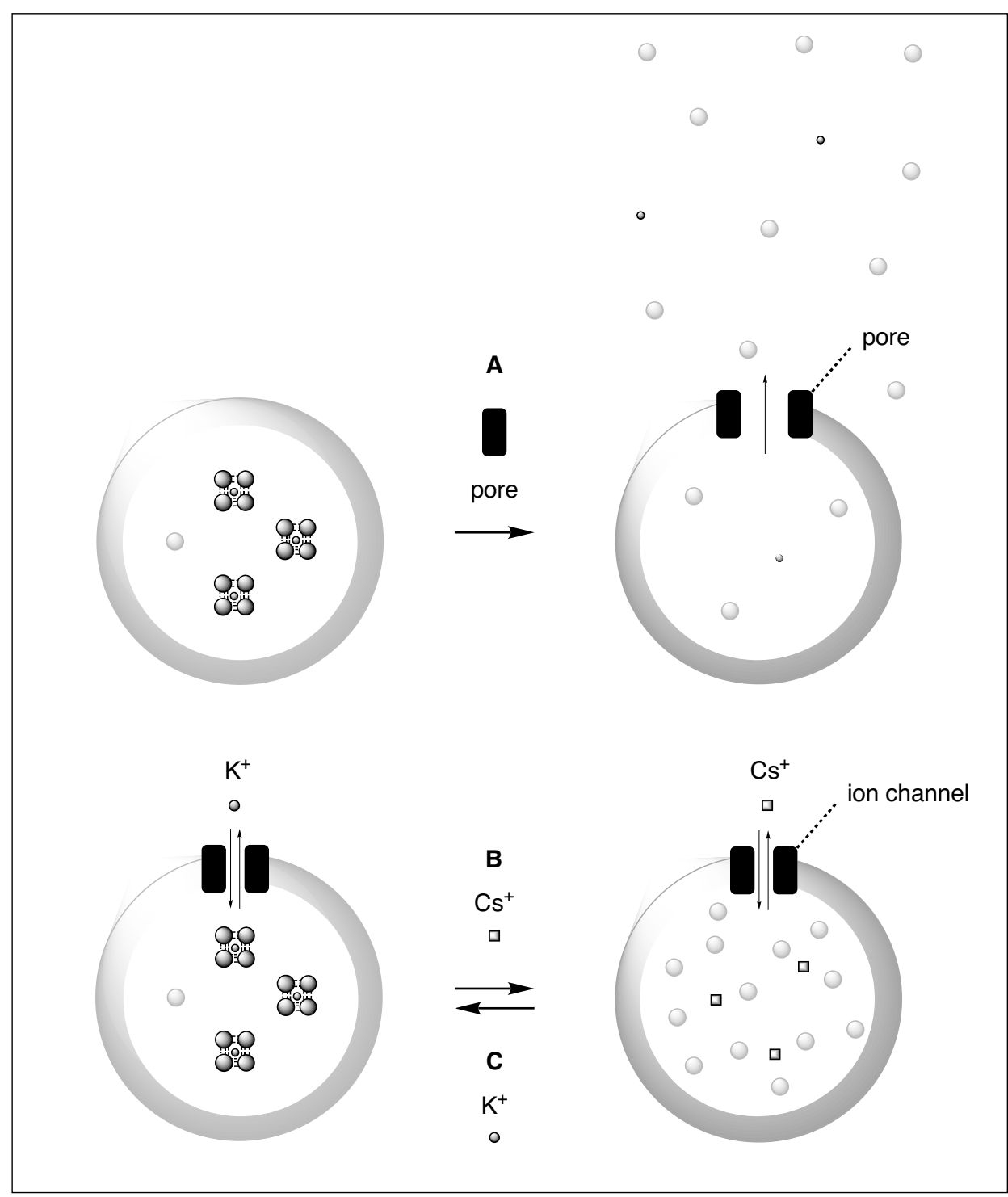

Fig. 3. Detection of the activity of pores $(A)$ and ion channels $(B, C)$ by $C D$ spectroscopy. (A) For the detection of pores by CD spectroscopy, vesicles are loaded with GMP and potassium cations at concentrations high enough to produce G-quartets within the vesicle. Addition of pores causes G-quartet disassembly due to GMP efflux. This is recorded as CD silencing. (B) For the detection of the activity of ion channels by CD silencing, the same vesicles with internal G-quartets are used. External $\mathrm{K}^{+} / \mathrm{Cs}^{+}$exchange followed by channel addition initiates transmembrane $\mathrm{Cs}^{+} / \mathrm{K}^{+}$exchange to disassemble the G-quartets within the vesicle. (C) The complementary chirogenic detection of the activity of ion channels uses vesicles loaded with GMP and $\mathrm{Cs}^{+}$instead of $\mathrm{K}^{+}$. External $\mathrm{Cs}^{+} /$ $\mathrm{K}^{+}$exchange followed by channel addition initiates transmembrane $\mathrm{K}^{+} / \mathrm{Cs}^{+}$exchange to cause the assembly of G-quartets within the vesicle.

tivity. Or so it seems. In reality, the situation turns out to be more complex. Many examples exist where otherwise confirmed supramolecular function gives a Hill coefficient of $n=1$ or even $n<1 .{ }^{[11,15]}$ Particularly the latter values clearly underscore contributions of phenomena beyond cooperativity to the observed Hill coefficient.

What is the cause of this confusing outcome? Significant contributors to the Hill coefficients are readily deducible from the Hill equation. Regen, and others, pointed out early on that the Hill equation is only valid when relevant monomer concentrations $c_{\mathrm{M}}$ are used.[16] Applied to the selfassembly of these monomers into an active supramolecule, this observation suggests that the Hill equation is compatible only with endergonic self-assembly. For the detection of supramolecular function, this implies that Hill coefficients $n>1$ are observed only for thermodynamically unstable supramolecular architectures. The conclusion is that Hill coefficients $n>1$ not only provide experimental evidence for active supramolecules but also demonstrate that the structure of these active supramolecules cannot be studied by routine methods because they exist as a minor population besides a large population of inactive monomers. In other words, with $n>1$ systems, as sometimes in real life, only a few do the real work while others just stand nearby and watch, and much attention, expertise and equipment is

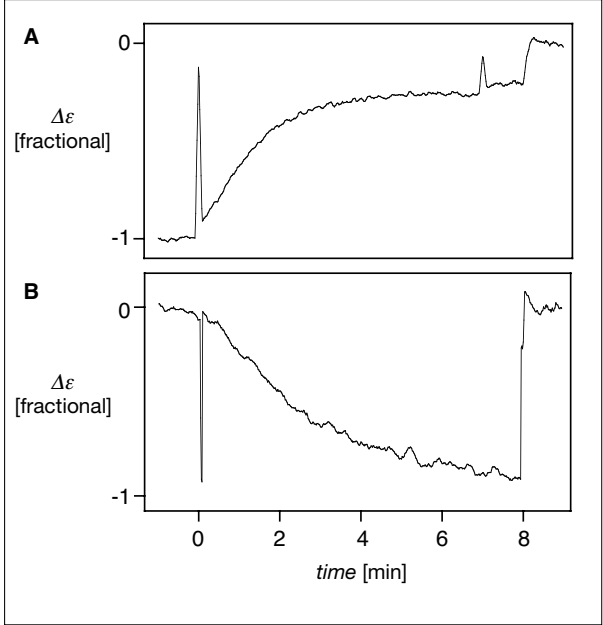

Fig. 4. Detection of the activity of ion channels as $C D$ silencing $(A)$ or chirogenesis $(B$, compare Fig. 3). (A) Change in $\Delta \varepsilon$ at $265 \mathrm{~nm}$ during the addition of gramicidin ( $0 \mathrm{~min}$ ) and detergent (8 min) to vesicles with internal GMP and $\mathrm{K}^{+}$and external $\mathrm{Cs}^{+}$. (B) Same with internal $\mathrm{Cs}^{+}$and external $\mathrm{K}^{+}$. Reproduced with permission from ref [10], (c) 2008 Wiley-Liss, Inc

needed to really zoom in and catch an image of the true action.

Hill analysis of stable active supramolecular architectures, on the other hand, should give $n=1$ without the evidence of cooperativity. In the frequent case of competing processes at higher concentrations of stable active supramolecules (such as precipitation), a Hill coefficient $n<1$ will make it even look like less than one molecule could do the job.

In other words, the outcome of functional Hill analysis is always unsatisfactory: Either i) experimental evidence for functional relevance is obtained for active supramolecules that are undetectable by routine structure determination $(n>1)$, or ii) experimental evidence for functional relevance is inaccessible for active supramolecules that are very well detectable by routine structure determination $(n \leq 1)$. To overcome this dilemma, we thought to artificially destabilize a suspected stable active supramolecule before Hill analysis by chemical denaturation (Fig. 5A). This should increase $c_{\mathrm{v}}$, restore compatibility with the Hill equation and confirm the suspected cooperativity with $n>1$ (Fig. 5B).

Rigid-rod molecule $\mathbf{3}$ offered an ideal occasion to test the validity of this denaturation approach to the identification of supramolecular function. ${ }^{[2,11]}$ It is a $p$-octiphenyl that carries eight naphthalenediimides (NDIs) along the scaffold. Rod $\mathbf{3}$ and its analog with blue NDI chromophores self-assemble into tetrameric rigid-rod $\pi$-stack architectures with ion channel and photosynthetic activity. ${ }^{[2,17-19]}$ The Hill analysis of the activity for the latter gave the expected $n \approx 4$. $^{[2]}$ The CD spectra of rod 


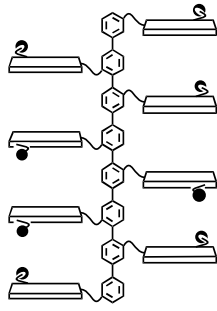

(A) denaturation

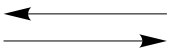

(B) Hill plot

monomer

3

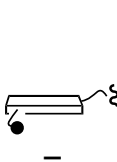

$=$

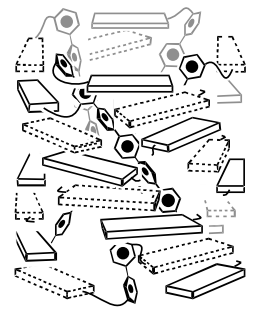

supramolecule

4

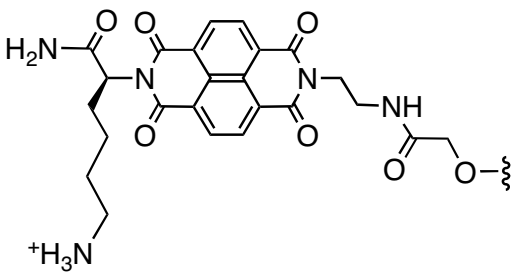

Fig. 5. Denaturation-corrected Hill analysis to identify existence and stoichiometry of stable supramolecules under conditions relevant for function, exemplified with the self-assembly of monomer $\mathbf{3}$ and analogs into supramolecules such as 4 . Chemical denaturation (A) before Hill analysis $(B)$ is applied to assure the high monomer concentrations needed for compatibility of Hill analysis with exergonic self-assembly. ${ }^{[11]}$

3 and analogs were in agreement with $M$ helical self-organization (negative amplitude of exciton-coupled CD (ECCD) doublets). Moreover, $\mathrm{CD}$ silencing in response to ligand intercalation provided excellent structural support for the untwisting of the $M$-helices with photosynthetic activity into barrel-stave supramolecules with ion channel activity. ${ }^{[12,18]}$ The CD spectra of rod 3 and analogs were, however, concentration independent (Fig. 6A). [11] The $n=$ 1 obtained from structural studies by $\mathrm{CD}$ spectroscopy is incompatible with the $n>1$ obtained from functional studies. This contradiction nicely spotlights the importance to conduct structural studies of supramolecular architectures under conditions that are meaningful for function, and provides an excellent system to probe the validity of denaturation-corrected Hill analysis.

The result was in agreement with expectations. In the presence of denaturants such as urea or guanidinium chloride, ${ }^{[13]}$ the CD spectra of rod 3 became concentration dependent (Fig. 6B). Denaturationcorrected Hill analysis confirmed with $n \approx$ 4 the cooperativity determined in functional studies (Fig. 6C). These findings identified denaturation-corrected Hill analysis by $\mathrm{CD}$ spectroscopy as a valid method to identify stable supramolecules under conditions that are relevant for function.

In conclusion, we described recent $\mathrm{CD}$ methods development to measure osmotic pressure and the activity of ion channels and pores with G-quartets as new and versatile CD probes, and to identify supra- molecules in action (and to determine Hill coefficients for exergonic self-assembly). These examples demonstrate the usefulness of chirality to study supramolecular function in a sensitive and selective manner under meaningful conditions. The potential for future $\mathrm{CD}$ methods development in this area is significant and deserves more scientific attention.

\section{Acknowledgements}

Financial support of this research by the Swiss NSF and the University of Geneva is gratefully acknowledged.

Received: February 15, 2008

[1] N. Sakai, J. Mareda, S. Matile, Acc. Chem. Res. 2005, 38, 79.

[2] S. Bhosale, A. L. Sisson, P. Talukdar, A. Fürstenberg, N. Banerji, E. Vauthey, G. Bollot, J. Mareda, C. Röger, F. Würthner, N. Sakai, S. Matile, Science 2006, 313, 84.

[3] S. Litvinchuk, H. Tanaka, T. Miyatake, D. Pasini, T. Tanaka, G. Bollot, J. Mareda, S. Matile, Nat. Mater. 2007, 6, 576.

[4] N. Sakai, A. L. Sisson, T. Bürgi, S. Matile, J. Am. Chem. Soc. 2007, 129, 15758.

[5] V. Gorteau, G. Bollot, J. Mareda, A. Perez-Velasco, S. Matile, J. Am. Chem. Soc. 2006, 128, 14788.

[6] T. Takeuchi, M. Kosuge, A. Tadokoro, Y. Sugiura, M. Nishi, M. Kawata, N. Sakai, S. Matile, S. Futaki, ACS Chem. Biol. 2006, 1, 299.

[7] J. Zhang, M. T. Albelda, Y. Liu, J. W. Canary, Chirality 2005, 17, 404.

[8] H. Tanaka, S. Matile, Chirality 2008, 20, 307.

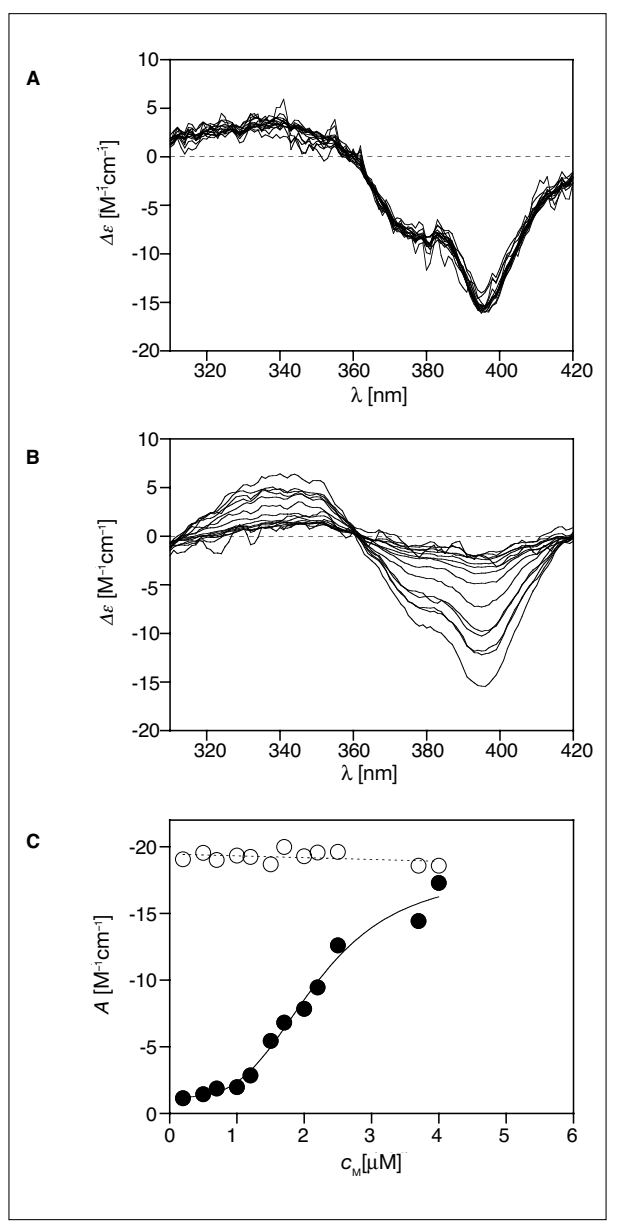

Fig. 6. CD spectra (A, B) and Hill plot (C) of monomer 3 near the detection limit in the absence $(\mathrm{A}, \mathrm{CO})$ and presence $(\mathrm{B}, \mathrm{C} \bullet)$ of denaturant (urea). CD amplitude $\mathrm{A}=\Delta \varepsilon_{1}(391 \mathrm{~nm})-\Delta \varepsilon_{2}(340$ $\mathrm{nm})$. Reproduced with permission from ref [11], (c) 2006 Wiley-Liss, Inc.

[9] N. Sakai, S. Matile, Chirality 2003, 15, 766.

[10] A. Hennig, S. Matile, Chirality, in press (DOI: 10.1002/chir.20526).

[11] S. Bhosale, S. Matile, Chirality 2006, 18, 849.

[12] N. Sakai, P. Talukdar, S. Matile, Chirality 2006, 18, 91 .

[13] G. Das, S. Matile, Chirality 2001, 13, 170.

[14] 'Circular Dichroism - Principles and Applications', Ed. K. Nakanishi, N. Berova, R. W. Woody, Weinheim, Wiley, 1994.

[15] S. Litvinchuk, G. Bollot, J. Mareda, A. Som, D. Ronan, M. R. Shah, P. Perrottet, N. Sakai, S. Matile, J. Am. Chem. Soc. 2004, 126, 10067.

[16] E. Stadler, P. Dedek, K. Yamashita, S L. Regen, J. Am. Chem. Soc. 1994, 116, 6677.

[17] P. Talukdar, G. Bollot, J. Mareda, N. Sakai, S. Matile, J. Am. Chem. Soc. 2005 127, 6528.

[18] P. Talukdar, G. Bollot, J. Mareda, N. Sakai, S. Matile, Chem. Eur. J. 2005, 11, 6525.

[19] S. Bhosale, A. L. Sisson, N. Sakai, S. Matile, Org. Biomol. Chem. 2006, 4, 3031. 